\title{
Extracorporeal Membrane Oxygenation Circuitry
}

\author{
Laurance Lequier, MD, FRCPC, \\ Director, ECMO Program, Stollery Children's Hospital, Associate Clinical Professor Pediatrics, \\ University of Alberta, Edmonton, AB, Canada
}

Stephen B. Horton, PhD, CCP(Aus), CCP(USA), FACBS, Associate Professor, Director of Perfusion, Faculty of Medicine, Department of Paediatrics, The University of Melbourne, Royal Children's Hospital, Melbourne, Australia

D. Michael McMullan, MD, FACS, and

Associate Professor of Surgery, Director, Mechanical Cardiac Support and ECMO, Seattle Children's Hospital, Seattle, WA

Robert H Bartlett, MD

Professor of Surgery, Emeritus, University of Michigan, Ann Arbor, MI

Laurance Lequier: laurance.lequier@albertahealthservices.ca

\begin{abstract}
The extracorporeal membrane oxygenation (ECMO) circuit is made of a number of components that have been customized to provide adequate tissue oxygen delivery in patients with severe cardiac and/or respiratory failure for a prolonged period of time (days to weeks). A standard ECMO circuit consists of a mechanical blood pump, gas exchange device, and a heat exchanger all connected together with circuit tubing. ECMO circuits can vary from simple to complex and may include a variety of blood flow and pressure monitors, continuous oxyhemoglobin saturation monitors, circuit access sites and a bridge connecting the venous access and arterial infusion limbs of the circuit. Significant technical advancements have been made in the equipment available for short and long term ECMO applications. Contemporary ECMO circuits have greater biocompatibility and allow for more prolonged cardiopulmonary support time, while minimizing the procedure-related complications of bleeding, thrombosis and other physiologic derangements that were so common with the early application of ECMO. Modern era ECMO circuitry and components are simpler, safer, more compact and can be used across a wide variety of patient sizes from neonates to adults.
\end{abstract}

\section{Keywords}

extracorporeal life support; pump; oxygenator; cannula

Extracorporeal membrane oxygenation (ECMO) is a modified form of cardiopulmonary bypass used to provide adequate tissue oxygen delivery in patients with severe cardiac and/ or respiratory failure. ECMO involves draining blood from the venous circulation, pumping

Correspondence to: Laurance Lequier, laurance. lequier@albertahealthservices. ca.

Publisher's Disclaimer: This is a PDF file of an unedited manuscript that has been accepted for publication. As a service to our customers we are providing this early version of the manuscript. The manuscript will undergo copyediting, typesetting, and review of the resulting proof before it is published in its final citable form. Please note that during the production process errors may be discovered which could affect the content, and all legal disclaimers that apply to the journal pertain.

Dr. Bartlett has received financial support from the National Institutes of Health. The authors have not disclosed any potential conflicts of interest. 
it through an artificial lung where oxygen is added and carbon dioxide is removed, and then returning the warmed blood back to either the venous or arterial circulation. ECMO and the associated management protocols will mechanically support a patient and allow for optimization of all aspects of care for the period of time necessary for recovery of native cardiac and/or respiratory function. The ECMO circuit consists of a number of components that have been customized to allow patients to be supported for a prolonged period of time (days to weeks), as opposed to a number of hours during cardiopulmonary bypass.

Significant technical advancements have been made in the equipment available for short and long term ECMO applications.

\section{ECMO Circuit Design}

A standard ECMO circuit consists of a mechanical blood pump, gas exchange device (membrane oxygenator), and a heat exchanger all connected together with circuit tubing between the venous access cannula and either the arterial (VA) or venous (VV) infusion cannula (Fig. 1) (1). Circuit tubing used is made from a polyvinylchloride (PVC) - based plastic compound. ECMO circuits can vary from simple to complex and may include a variety of blood flow and pressure monitors, continuous oxyhemoglobin saturation monitors, circuit access sites and a bridge connecting the venous access and arterial infusion limbs of the circuit. Blood is exposed to a large surface area as it moves through the ECMO circuit, causing significant heat loss, and children on ECMO require direct warming of the blood to maintain body temperature.

Individual centers customize their ECMO circuits to suit their patient population and program needs. In general, the less complicated the circuit, the easier it will be to manage and the fewer the connectors and stopcocks, the less potential sites of flow turbulence and blood stasis. ECMO circuits need to be functional and portable, yet ideally, one should attempt to limit the size of the foreign surface of the circuit. This is because when blood is exposed to the non-biologic surfaces of a circuit, a complex biologic response is initiated involving both the coagulation pathway and the inflammatory response pathway (2). The tubing of the circuit can be coated with a biocompatible lining to reduce the systemic inflammatory response and risk of thrombosis and bleeding (3), although no coating to date has been shown to eliminate this reaction completely. Shorter tubing may also optimize venous drainage, as there is less resistance to blood flow (4). ECMO circuits usually have a variety of monitors including an integrated blood pump flow monitor which measures total circuit blood flow, as well as separate ultrasonic flow detectors that can be placed on venous drainage and arterial infusion limbs of the circuit. The best way to monitor ECMO circuits is without interrupting the blood path. This means that flow, saturations, pressures and blood chemistry should ideally be measured without the inclusions of cuvettes, connectors or added tubing into the blood flow path. Circuit pressures can be measured at three important locations within the ECMO circuit. Venous access pressures measured before a centrifugal blood pump (see below) help ensure excessive suction is avoided and help determine the adequacy of venous drainage and circuit volume. Circuit pressures are also commonly measured before and after the gas exchange device and this is also where most centers have circuit access sites. If both pressures rise, this indicates increased resistance to flow postoxygenator and may be a result of obstruction to the inflow cannula. An increase in the difference between the pre- and post-oxygenator (transmembrane) pressures suggest an increase in the resistance inside the oxygenator. Oxyhemoglobin saturation of circuit blood is most useful on the venous access limb of the ECMO circuit, especially in VA support, to help assess the adequacy of oxygen delivery and support.

ECMO circuits can include a bridge of circuit tubing connecting the proximal venous access limb of the circuit to the proximal arterial infusion limb of the circuit. This bridge is used to 
recirculate blood through the circuit if the patient needs to be removed temporarily from support. It is also particularly useful in VA support during weaning of flow to low levels of support. The patient can be clamped off from the ECMO circuit to determine if adequate gas exchange and hemodynamics can be maintained while flow continues through the bridge. There are a number of modifications to the bridge that have been employed, including an open bridge (regulating flow with a thumb clamp), placing a clamp on the bridge to occlude flow totally, and a bridge that is closed with stopcocks which can be opened when needed (5). All forms of clamping, reopening and circulating through the bridge may increase turbulence in the system and disturb cerebral blood flow, especially when the patient is cannulated via the cervical vessels. Complete removal of the bridge is now favored in some centers.

ECMO circuits can be crystalloid-primed for use relatively quickly, and stored safely for up to 30 days (6). If time permits, most pediatric patients will receive a blood-primed ECMO circuit. The composition of the blood prime varies from center to center but usually contains a mixture of packed red blood cells, albumin, fresh frozen plasma, unfractionated heparin, and calcium to replace that bound by citrate in the banked blood.

\section{Pumps}

A pump is an essential component of the ECMO circuit. Semiocclusive roller pumps have been the standard for decades, but have mainly been replaced by novel centrifugal pumps. The ECMO pump must provide flow appropriate for the patient (typically $75-150 \mathrm{~mL} / \mathrm{kg}$ / min for infants and children), within a safe range of pressures to avoid hemolysis. Outlet pressure is a function of the pump speed and the resistance in the tubing, cannula, and arterial pressure of the patient. The risk of high outlet pressure is rupture of the circuit. The circuit should withstand pressure of $600 \mathrm{~mm} \mathrm{Hg}$, but $300 \mathrm{~mm} \mathrm{Hg}$ is considered the upper safe limit for most applications. The risk of low inlet pressure is hemolysis, which occurs when gas cavitates out of the blood (more than $600 \mathrm{~mm} \mathrm{Hg}$ suction). In roller pumps the suction is limited to the siphon from the patient to the pump (typically $100-150 \mathrm{~mm} \mathrm{Hg}$ ). Centrifugal pumps can generate $600 \mathrm{~mm} \mathrm{Hg}$ suction for brief periods whenever the drainage is occluded and RPMs are over 4000. Measuring inlet pressure closer to the pump gives a greater absolute pressure than by measuring at the cannula connection (4).

Roller pumps could generate direct suction on the venous catheter. In practice, this problem is avoided by the inclusion of a small collapsible bladder positioned at the lowest point of the venous line. The bladder (or a transducer directly in the venous line) is attached to an electrical switch that slows or stops the roller pump when a threshold suction is reached, then restarts the pump instantly when the filling pressure exceeds the pump suction (i.e., the venous drainage flow exceeds the pump flow). The suction on the venous cannula is the siphon created by the distance from the patient to the floor (typically $100-150 \mathrm{~cm} \mathrm{H}_{2} \mathrm{O}$ ). Whenever the bladder collapses (or the transducer senses subatmospheric pressure) and the pump stops, the suction effect of the siphon between the patient and the level of the bladder stops, avoiding any continuing suction on the right atrium. Also, because the pump motor is slowed or turned off whenever the bladder is collapsed, the pump cannot generate excessive suction in the blood between the pump and the bladder (which would cause cavitation and hemolysis). Thus, this bladder and electrical switching mechanism provides servo-regulation and some measure of safety for prolonged perfusion with a roller pump. The pump is adjusted to provide flow for the desired level of gas exchange or cardiac support. As long as the venous drainage is adequate, suction force is acceptable and the desired flow is delivered. If venous drainage is impeded for any reason (eg. hypovolemia, pneumothorax, kinking of the venous catheter), the pump stops and an alarm sounds. Flow resumes as soon as venous drainage is reestablished. Early in the course of extracorporeal circulation, the 
operator increases the flow to the point at which the pump is stopped by servo-regulation, thus identifying the physical limitation of venous drainage for the system. This flow rate is usually considerably greater than the flow actually required for extracorporeal support. However, if maximal flow through the system is inadequate after improving volume status, the venous catheter must be assessed. This may result in attempts to manipulate catheter position, replace it with a different design or larger cannula, or add a second catheter to gain more flow. The problems with roller pumps are: a sizable heavy motor is required, the tubing can wear or rupture in the pump head, and there is no limit to infusion pressure so there is always a risk of blowout.

Centrifugal pumps, in which a spinning rotor generates flow and pressure, are replacing roller pumps in many centers. New models have long pump head durability and can be used for prolonged extracorporeal circulation. Unlike roller pumps, the motor can be light and small, the components do not wear out, and the perfusion pressure is limited by the revolutions per minute (RPM), so return line pressure is low and circuit rupture is extremely rare. In long term ECMO, or prolonged in-vivo experiments, a superiority in blood-handling characteristics has been identified with centrifugal pumps, as long as inlet pressures are monitored and do not become excessive (7). The potential problems of a centrifugal pump head include stagnation and heating in the pump head, leading to thrombus at low flows or if the outlet line is occluded, and cavitation and hemolysis when the inlet line is occluded. New pump head designs have reduced the magnitude of many of these problems. Mendler and colleagues initially introduced the Maquet Rotaflow pump (Maquet, Hirrlingen, Germany) as a new centrifugal pump in 1995. This pump was able to reduce heat generation of the bearing and seal, and improve hydraulic efficiency with less ensuing blood damage than other commercially available devices (8). These and other novel centrifugal pumps for long term use have a hole in the center of the rotor which solves the stagnation, thrombosis, and heat problems with older centrifugal pumps. When centrifugal pumps are used for cardiac surgery, the pump attaches directly to the venous reservoir and servo-regulation is provided by level sensing or by the pump operator. In ECMO, the pump inlet line is attached directly to the venous cannula. Many times a day, the venous drainage may be transiently but completely occluded (e.g., during coughing, hypovolemia, or kinking, manifested as "chattering" of the venous line). When the venous line is occluded, the rotor keeps spinning, evacuating blood from the pump head and creating a vacuum in the pump head which causes cavitation and hemolysis. This happens in a second (milliseconds at high RPM), so no servo-regulation system is fast enough to prevent cavitation. The problem can be minimized by incorporating a collapsible bladder, such as the Better Bladder (Circulatory Technology, Oyster Bay, NY), in the venous line to act as a mini reservoir by servo-regulating pump RPM based on inlet pressure sensing, which prevents continuing suction when the line is occluded for more than a few seconds (9). However, this adds further complexity and prime volume to the circuit. In practice, many centers deal with the problem by limiting the amount of RPM and reducing the flow when the venous line is chattering. Novel centrifugal pumps such as Maquet Rotaflow (Maquet) and Cobe Revolution (Cobe Cardiovascular, Inc., Arvada, $\mathrm{CO}$ ) have small priming volumes and minimize thrombus formation, heat generation and hemolysis associated with traditional centrifugal pumps (10). Hemolysis may be caused by heat generation and thrombus formation in the pump head, stagnant or turbulent blood flow zones in the pump head, oxygenator or other places in the circuit, shear stress caused by high blood flow velocities, excessive suction, and circuit thrombosis. The Thoratec Centrimag pump (Thoratec, Pleasanton, CA) features bearing-less technology; the rotor is levitated into the housing by the magnetic force generated by the motor, hence minimizing friction and improving hemocompatibility. The risk of thrombus formation is reduced by uniform unidirectional flow and less stagnation, while reduced shearing stress attenuates hemolysis. A number of studies have shown a reduction in circuit-related complications and hemolysis when comparing the use of novel centrifugal pumps to either 
roller pumps or traditional centrifugal pumps $(11,12)$. Other reports have found increased hemolysis and renal failure with centrifugal systems, although these studies included older centrifugal systems and were not focused on newer devices (13).

Complete ECMO pump systems are available from a number of commercial vendors including: Maquet (Hirrlingen, Germany), Medos (Stolberg, Germany), Sorin (Mirandola, Modena, Italy) and Thoratec (Pleasanton, CA). All use a modified centrifugal pump, are theoretically safer, more portable and some contain a complete ECMO system with all necessary components in an integrated, more compact product.

\section{Oxygenators}

ECMO circuits have a gas exchange device called an oxygenator, to add $\mathrm{O}_{2}$ and remove $\mathrm{CO}_{2}$ from blood. It may contain several different biomaterials including silicone rubber as in the classic membrane lungs used for years, polypropylene hollow fiber devices for short term use, newer compressed surface polymethylpentene (PMP), as well as polyvinylchloride, polyurethane and stainless steel. The surface area and blood path mixing determine the maximum oxygenation capacity of any gas exchange device. The rated flow of an oxygenator is defined by the amount of desaturated (75\%) blood that can be nearly fully saturated (95\%) per minute. The Kolobow silicone rubber membrane lung has been the standard oxygenator used for ECMO applications for almost 50 years (14). It is constructed of a flat reinforced sheet of silicone rubber membrane envelope wrapped around a wire mesh in a spiral coil. Blood and gas flow in counter-current directions within the silicone lung and gas exchange occurs by diffusion across the membrane. This membrane oxygenator is very effective at exchanging $\mathrm{O}_{2}$ and $\mathrm{CO}_{2}$, but it is necessary to have a variety of sizes available to support different size patients. A separate heat exchanger is required for smaller silicone membrane lungs. The silicone lung has a high resistance to flow which limits the maximum blood flow that could be otherwise obtained through the device and makes them less suitable for use with centrifugal pumps. By nature of its design and high resistance a silicon oxygenator is much harder to de-air, so it takes longer to prime and is more difficult for transports. Despite these limitations, the Kolobow membrane lung and roller pump served the needs of the ECMO community for decades.

Hollow fiber PMP oxygenators are extremely efficient at gas exchange and demonstrate minimal plasma leakage; have relatively low resistance to blood flow, making them easy to prime; and are well suited for use with centrifugal blood pumps (Fig. 2) (15). The design for bundling the hollow fibers with gas inside and blood outside the fibers within the oxygenator, the winding technique used, and the blood flow pattern through the device are all key factors in decreasing the priming volume. By making the oxygenators more compact and optimizing the blood flow path, it is possible to decrease the surface area of the membrane and heat exchanger, thus reducing its potential for thrombus formation and inflammatory activation. The low prime volume enables a center to utilize one device for all size of patients and these circuits can be left assembled and crystalloid-primed, with the benefit of support implementation within minutes. The early experience with the PMP devices established them to be robust and long-lasting, with limitation of the inflammatory response and decreased transfusion requirements, making them well suited for long-term use (16). There are a number of PMP membrane oxygenators in commercial use including the Quadrox-iD (Maquet, Hirrlingen, Germany), Hilite LT (Medos, Stolberg, Germany), Lilliput 2 (Sorin, Mirandola Modena, Italy), and the Biocube (Nipro, Osaka, Japan). Many of these devices are marketed in both pediatric and adult sizes; however, many centers prefer to use one size of oxygenator for all patients. These new generation oxygenators also contain an integrated heat exchange device, making it possible to precisely control body temperature without the need for additional components. 


\section{Vascular Cannulas}

Like other components of the modern ECMO circuit, vascular cannulas have undergone significant design changes which have improved efficiency of blood flow and overall performance. Several types of cannulas are currently available in a variety of sizes, with distinct features that may be used to adopt a cannulation strategy that is customized to the unique requirements of individual patients. Single-lumen cannulasare used to provide venous and arterial access for patients receiving VA ECMO or multiple site venous access for patients receiving VV extracorporeal support. Most cannulas are manufactured from biocompatible polyurethane, which may be coated with heparin or non-heparin polymers that may reduce platelet activation and the inflammatory response at the blood-cannula interface (17). Cannulas are available in sizes ranging from $6 \mathrm{~F}(2 \mathrm{~mm}$ diameter) to $51 \mathrm{~F}$ (17 $\mathrm{mm}$ diameter). Most cannulas are manufactured with wire-reinforced bodies that are designed to prevent luminal occlusion. In addition, some incorporate a malleable wire along the length of the cannula wall, which may be used to customize the angle of insertion and position within the heart when used during open-chest ECMO. Various cannula tip configurations are available. Right-angle metal tip cannulas, commonly used for venous drainage during cardiopulmonary bypass, provide excellent right atrial drainage during postcardiotomy support. Cannulas that employ a long, multi-fenestrated flexible tip are useful for jugular and femoral venous drainage, whereas cannulas that contain a single end-hole or a short fenestrated tip may be used for arterial or venous vascular access. Many cannulas are designed for percutaneous vascular access using cannula-specific guide wire introducer sets. Overall cannula length and cross-sectional area, which impart an inherent resistance to blood flow, must be considered during cannula selection to achieve optimal venous drainage.

Consequently, cannula selection should be based on the estimated level of support (flow rate) to be provided and the size of the vessels to be accessed.

Dual-lumen cannulas provide venovenous support via a single jugular venous access site. Blood is removed from the patient via one lumen and then returned to the patient via a smaller lumen. There are currently three commercially available dual-lumen ECMO cannulas. The OriGen (Austin, TX) dual-lumen cannula is available in $12 \mathrm{~F}, 15 \mathrm{~F}$, and $18 \mathrm{~F}$ sizes and capable of providing extracorporeal gas exchange for patients up to $12 \mathrm{~kg}$. The OriGen cannula may be inserted percutaneously using a guide wire or directly into the internal jugular vein through an incision in the overlying soft tissues. Proper positioning of the tip of the cannula near the inferior cavoatrial junction is necessary for optimal cannula performance. Blood drainage and return ports are spatially separated to decrease recirculation of blood within the ECMO circuit, which reduces overall efficiency of gas exchange (18). A significant limitation of the OriGen cannula is that it is manufactured from non-wire-reinforced polyurethane that is subject to structural deformation after insertion. Kinking and collapse of the cannula caused by excessive suction can result in catastrophic interruption of ECMO flow. Refinement of the catheter to alleviate this problem is underway. The Covidien ECMO (Mansfield, MA) cannula is also manufactured from nonwire-reinforced polyurethane. It is only available in $14 \mathrm{~F}$ overall diameter, limiting its use to neonatal patients with respiratory failure. The Avalon (Rancho Dominguez, CA) Bi-Caval dual-lumen cannula is manufactured from a wire-reinforced silicone polyurethane polymer that resists structural deformation. It may be inserted through the internal jugular vein via open surgical or percutaneous techniques. A unique feature of the Avalon cannula is that, when properly inserted, the distal tip is placed in the inferior vena cava, which facilitates simultaneous removal of blood from the superior and inferior vena cavae and return of blood to the right atrium. Echocardiographic or fluoroscopic imaging is necessary to verify proper cannula placement. The Avalon cannula is available in sizes ranging from $13 \mathrm{~F}$ to $31 \mathrm{~F}$, which enables it to be used to support neonates through to adults. However, some centers have 
reported delayed atrial perforation when using the $13 \mathrm{~F}$ cannulas, which may limit use of the Avalon cannula to older infants, children, and adults.

\section{Interhospital Transport Circuits}

The growth in the field of ECMO transport is one that parallels that of other ECMO circuitry. Specific circuits containing smaller, more biocompatible components have expanded the possibilities of patient transport on ECMO. Circuit simplification utilizing PMP membrane oxygenators and centrifugal pumps has allowed safer movement of patients from facilities that do not provide ECMO to those equipped with the expertise to support patients for extended periods. The field of transportation continues to evolve with purposebuilt transport systems such as the Cardiohelp (Maquet, Hirrlingen, Germany) (19), further simplifying the setup and monitoring of patients moving around or in between hospitals.

\section{Conclusion}

Modern era ECMO circuitry and components are simpler, safer, more compact and can be used across a wide variety of patient sizes from neonates to adults. Today's ECMO circuits have greater biocompatibility and allow for more prolonged cardiopulmonary support time while minimizing procedure-related complications of bleeding, thrombosis and other physiologic derangements that were so common with the early application of ECMO.

\section{References}

1. Toomasian, JM.; Lawson, S.; Harris, WE. The Circuit. In: Annich, GM.; Lynch, WR.; MacLaren, G.; Wilson, JM.; Bartlett, RH., editors. Extracorporeal Cardiopulmonary Support in Critical Care. 4. Extracorporeal Life Support Organization; 2012. p. 107-132.

2. Peek GJ, Firmin RK. The inflammatory and coagulative response to prolonged extracorporeal membrane oxygenation. ASAIO J. 1999; 45(4):250-63. [PubMed: 10445729]

3. McMullan DM, Emmert JA, Permut LC, et al. The inflammatory and coagulative response to prolonged extracorporeal membrane oxygenation. Eur J Cardiothorac surg. 2011; 39:392-397. [PubMed: 20801051]

4. Augustin S, Horton A, Butt W, Bennett M, Horton S. Centrifugal pump inlet pressure site affects measurement. Perfusion. 2010; 25:313-320. [PubMed: 20630918]

5. Totapally BR, Sussmane JB, Hultquist K, et al. Variability in systemic arterial pressure during closed- and open-bridge extracorporeal life support: an in vitro evaluation. Crit Care Med. 2000; 28(6):2076-80. [PubMed: 10890667]

6. Walczak R, Lawson DS, Kaemmer D, et al. Evaluation of a preprimed microporous hollow-fiber membrane for rapid response neonatal extracorporeal membrane oxygenation. Perfusion. 2005; 20:269-275. [PubMed: 16231623]

7. Horton A, Butt W. Pump-induced hemolysis: is the constrained vortex pump better or worse than the roller pump? Perfusion. 1992; 7:103-108.

8. Mendler N, Podecht F, Feil G, Hiltmann P, Sebening F. Seal-less centrifugal blood pump with magnetically suspended rotor: rot-a-flot. Artificial Organs. 1995; 19(7):620-4. [PubMed: 8572962]

9. Tamari Y, Lee-Sensiba K, King S, Hall MH. An improved bladder for pump control during ECMO procedures. J Extra Corpor Technol. 1999; 31:84-90. [PubMed: 10724648]

10. Lawson DS, Ing R, Cheifetz IM, et al. Hemolytic characteristics of three commercially available centrifugal blood pumps. Ped Crit Care Med. 2005; 6:573-577.

11. Byrnes J, McKamie W, Swearingen C, et al. Hemolysis during cardiac extracorporeal membrane oxygenation: A case control comparison of roller umps and centrifugal pumps in a pediatric population. ASAIO J. 2011; 57:456-461. [PubMed: 21822124]

12. Yu K, Long C, Hei F, et al. Clinical Evaluation of Two Different ECMO Systems: A Single Center Report. Artif Organs. 2011; 35:733-737. [PubMed: 21375546] 
13. Barrett CS, Jaggers JJ, Cook EF, et al. Outcomes of neonates undergoing extracorporeal membrane oxygenation support using centrifugal versus roller blood pumps. Ann Thorac Surg. 2012; 94:1635-41. [PubMed: 22921236]

14. Kolobow T, Bowman RL. Construction and evaluation of an alveolar membrane heart lung. Trans Am Soc Artif Intern Organs. 1963; 9:238-245. [PubMed: 14034415]

15. Horton S, Thuys C, Bennett M, Augustin S, Rosenberg M, Brizard C. Experience with the JostraRotaflow and QuadroxD oxygenator for ECMO. Perfusion. 2004; 19:17-23. [PubMed: 15072251]

16. Peek GJ, Killer HM, Reeves R, Sosnowski AW, Firmin RK. Early experience with a polymethyl pentene oxygenator for adult extracorporeal life support. ASAIO J. 2002; 48(5):480-2. [PubMed: 12296566]

17. Mangoush O, Purkayastha S, Haj-Yahia S, et al. Heparin-bonded circuits versus nonheparinbonded circuits: an evaluation of their effect on clinical outcomes. Eur J Cardiothorac Surg. 2007; 31:1058-69. [PubMed: 17306555]

18. Rais-Bahrami K, Walton DM, Sell JE, Rivera O, Mikesell GT, Short BL. Improved oxygenation with reduced recirculation during venovenous ECMO: comparison of two catheters. Perfusion. 2002; 17:415-9. [PubMed: 12470030]

19. Haneya A, Philipp A, Foltan M, Camboni D, Müeller T, Bein T, Schmid C, Lubnow M. First experience with the new portable extracorporeal membrane oxygenation system Cardiohelp for severe respiratory failure in adults. Perfusion. 2012; 27:150-5. [PubMed: 22249962] 


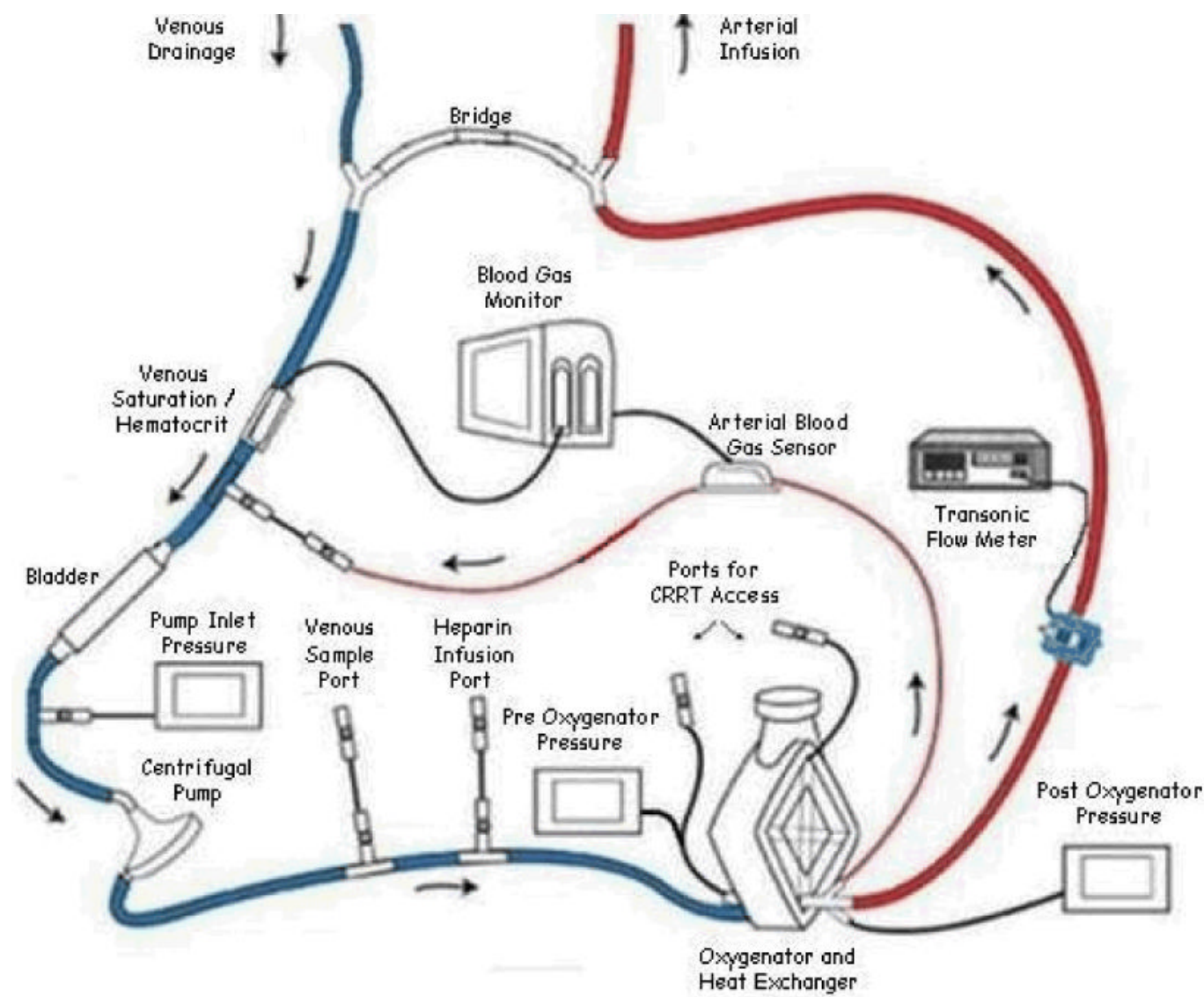

Figure 1.

Standard ECMO circuit. Venous blood drains from the patient, passes through a venous saturation sensor and a bladder before being pumped to the oxygenator/heat exchanger device. The oxygenated, warmed blood passes the ECMO circuit bridge before infusing back into the patient into the arterial (VA) or venous (VV) system. There are multiple infusion and access ports as well as pressure and flow monitors along the way. Adapted from (1). 


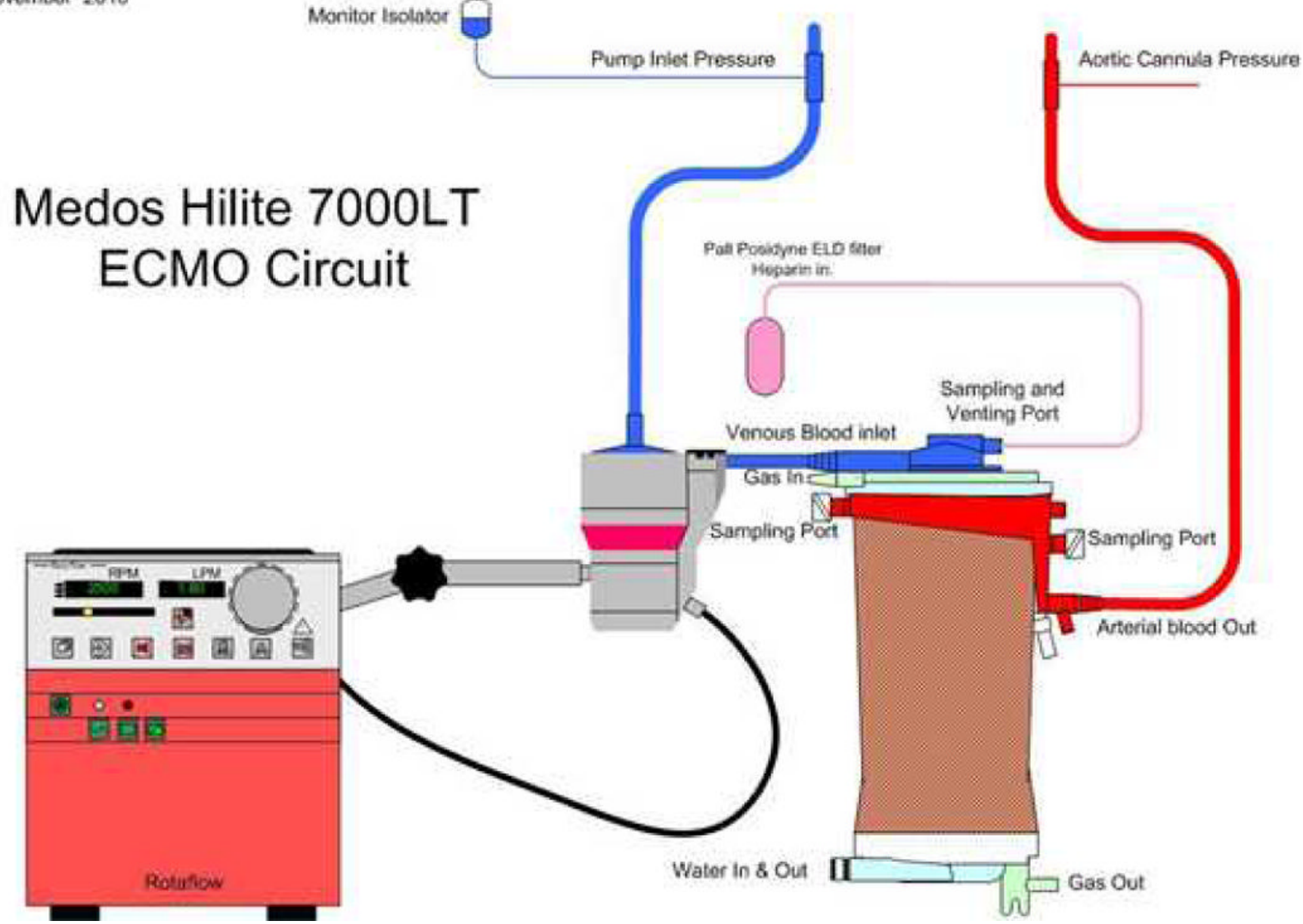

Figure 2.

Contemporary ECMO Circuit. This circuit has been improved over by the years and incorporates a radial blood pump, a PMP oxygenator and a simple minimal circuit. Adapted from (15) 